\title{
THE DENSEST LATTICE PACKING OF TETRAHEDRA
}

\author{
BY DOUGLAS J. HOYLMAN ${ }^{1}$
}

Communicated by Victor Klee, May 21, 1969

The problem of finding the densest packing of tetrahedra was first suggested by Hilbert [3, p. 319]. Minkowski [4] attempted to find the densest lattice packing of tetrahedra, but his result is invalid due to the incorrect assumption that the difference body of a regular tetrahedron was a regular octahedron. A lower bound for the maximum density of such a packing has been given by Groemer [1] as $18 / 49$. The purpose of this paper is to announce the proof that $18 / 49$ is in fact the maximum possible density.

We shall use the term convex body to mean any compact, convex set in three dimensions with nonempty interior, and lattice to mean the collection of all points (vectors) $m A+n B+p C$, where $m, n, p$ range over all integers and $A, B, C$ are three fixed linearly independent vectors. If $J$ is a convex body and $\Lambda$ is a lattice such that, when $x$ and $y$ are distinct points of $\Lambda$, the bodies $x+J$ and $y+J$ have no interior points in common, then the collection of bodies $\Lambda+J$ $=\{x+J: x \in \Lambda\}$ is said to form a lattice packing. If $\Delta(\Lambda)$ $=|\operatorname{det}(A, B, C)|$, and $\operatorname{Vol}(J)$ represents the volume of $J$, then the density of the packing is defined to be $\operatorname{Vol}(J) / \Delta(\Lambda)$. Minkowski [4] showed that $\Lambda+J$ is a lattice packing if and only if there are no points of $\Lambda$ other than the origin in the interior of the difference body $J-J$ $=\{x-y: x, y \in J\}$. When the latter condition holds, $\Lambda$ is said to be admissible for the difference body. A lattice is critical for a difference body if it is admissible and if no other admissible lattice has a smaller determinant. It follows that the problem of finding the densest lattice packing for a given convex body $J$ is equivalent to that of finding a critical lattice for $J-J$. The following lemmas are also from Minkowski's paper.

Lemma 1. If $\{A, B, C\}$ is a basis for the lattice $\Lambda$, if $A, B, C$ are on the boundary of the difference body $R$, and if none of the lattice points $A \pm B, A \pm C, B \pm C, A \pm B \pm C, 2 A \pm B \pm C, A \pm 2 B \pm C, A \pm B \pm 2 C$ is interior to $R$, then $\Lambda$ is admissible for $R$.

Lemma 2. If $\Lambda$ is a critical lattice for the difference body $R$, then $\Lambda$ has a basis $\{A, B, C\}$ such that $A, B, C$, and either

1 This paper is based upon a doctoral dissertation submitted to the University of Arizona. 
(I) $A-B, B-C$, and $C-A$ or

(II) $A+B, B+C$, and $C+A$

all lie on the boundary of $R$.

These results indicate the method for finding a critical lattice: we consider all lattices meeting the conditions of Lemma 2 and such that none of the lattice points listed in Lemma 1 is interior to the difference body, and find the one with the smallest determinant.

In particular, we consider the tetrahedron $T$ with the vertices $(-1,1,1),(1,-1,1),(1,1,-1)$ and $(-1,-1,-1)$. It can then be shown that the difference body of $T$ is the cubooctahedron $K$ described by

$|x| \leqq 2, \quad|y| \leqq 2, \quad|z| \leqq 2$, and $\quad|x|+|y|+|z| \leqq 4$

(see Hancock [2] or Groemer [1]). $K$ has six square faces and eight triangular faces. The problem may now be divided into cases according to the distribution of the basis vectors $A, B, C$ among the faces of $K$. It is easily shown that no two points of an admissible lattice can lie within the same triangular face of $K$. Using this fact and the symmetries of $K$, we find nineteen essentially different ways to assign $A, B$ and $C$ to the faces of $K$. Combining each of these with (I) or (II) from Lemma 2, we obtain thirty-eight cases. By means of computations which are much too long to reproduce here, it can be shown that in none of these cases can we obtain a lattice meeting the above conditions with a determinant less than $196 / 27$. This determinant is that of the lattice with basis

$A=(2,-1 / 3,-1 / 3), \quad B=(-1 / 3,2,-1 / 3), \quad C=(-1 / 3,-1 / 3,2)$,

which consequently is a critical lattice for $K$. Since $\operatorname{Vol}(T)$ is $8 / 3$, we obtain

THEOREM 1. The greatest possible density for a lattice packing of the regular tetrahedron $T$ is $18 / 49$; furthermore, the lattice which gives this packing is unique up to reflections in the coordinate planes, rotations of $90^{\circ}$ about any of the coordinate axes, and combinations of these.

Since any tetrahedron may be taken into $T$ by an appropriate nonsingular affine transformation, and since such a transformation does not change either the property of being a lattice packing or the density of a lattice packing, we have

COROLLARY. The greatest possible density for a lattice packing of any tetrahedron is $18 / 49$. 
If a convex body is symmetric about the origin, then its difference body is obtained by multiplying each vector in the given body by 2 . In particular, the difference body of $\frac{1}{2} K$ is $K$, so that the critical lattice for $K$ given above will yield the densest lattice packing of $\frac{1}{2} K$ as well. Since $\operatorname{Vol}\left(\frac{1}{2} K\right)=20 / 3$, we obtain

THEOREM 2. The greatest possible density for a lattice packing of cubooctahedra is 45/49; furthermore, the lattice which gives this packing is unique up to reflections in the planes of symmetry of the cubooctahedron, rotations of $90^{\circ}$ about its axes of symmetry, and combinations of these.

\section{REFERENCES}

1. H. Groemer, Über die dichteste gitterförmige Lagerung kongruenter Tetraeder, Monatsh. Math. 66 (1962), 12-15.

2. H. Hancock, Development of the Minkowski geometry of numbers, Macmillan, New York, 1939.

3. D. Hilbert, "Mathematische Probleme", Archiv. Math. Phys. 3 (1901), no. 1 44-63; 213-237, in Gesammelte Abhandlungen. Vol. III, Chelsea, New York, 1965, pp. 290-329.

4. H. Minkowski, "Dichteste gitterförmige Lagerung kongruenter Körper", Nachr. K. Ges. Wiss. Göttingen, 1904, 311-355, in Gesammelte Abhandlungen. Vol. II, Teubner, Berlin, 1911, pp. 3-42.

University of Arizona, Tucson, Arizona 85721 